\title{
Corps de femmes, corps dociles : le cas Magdalen Laundries
}

Nathalie Sebbane

\section{(2) OpenEdition \\ 1 Journals}

Édition électronique

URL : http://journals.openedition.org/etudesirlandaises/5082

DOI : 10.4000/etudesirlandaises. 5082

ISSN : 2259-8863

\section{Éditeur}

Presses universitaires de Rennes

\section{Édition imprimée}

Date de publication : 29 juin 2017

Pagination : 17-30

ISBN : 978-2-7535-5495-5

ISSN : 0183-973X

\section{Référence électronique}

Nathalie Sebbane, "Corps de femmes, corps dociles : le cas Magdalen Laundries », Études irlandaises [En ligne], 42-1 | 2017, mis en ligne le 29 juin 2019, consulté le 06 septembre 2019. URL : http:// journals.openedition.org/etudesirlandaises/5082 ; DOI : 10.4000/etudesirlandaises.5082 


\title{
Corps de femmes, corps dociles : le cas Magdalen Laundries
}

\author{
Nathalie Sebbane \\ Université Sorbonne Nouvelle-Paris 3
}

\section{Résumé}

Alors que l'année 2016 marque le $20^{\mathrm{e}}$ anniversaire de la fermeture de la dernière Magdalen Laundry en Irlande, cet article offre une perspective historique sur la manière dont ces institutions, s'inscrivant dans le schéma de la logique foucaldien du grand renfermement, ont caché, discipliné et contrôlé les corps des femmes qui contrevenaient à l'ordre moral d'abord au dixneuvième siècle puis surtout après la naissance de l'État Libre.

Mots clés : Magdalen Laundries, femmes, corps, institutions, discipline.

\section{Abstract}

The year 2016, commemorating the 100 th anniversary of the Easter Rising, also marked the $20^{\text {th }}$ anniversary of the closing of the last Irish Magdalen Laundry. This paper offers a historical analysis of the way these institutions were part of a broader logic of internment of transgressive individuals, whose aim was to conceal and control women's bodies. It shows that in Ireland, specific techniques were used in order to perform this taming of the body, in a context of identity construction after 1922.

Keywords: Magdalen Laundries, women, body, institutions, discipline.

Si l'année 2016 s'inscrit dans une décennie de commémorations en Irlande avec le centenaire du soulèvement de Pâques 1916, elle a également marqué le $20^{\mathrm{e}}$ anniversaire de la fermeture de la dernière Magdalen Laundry, institution conventuelle de prise en charge des femmes dites "perdues ", à savoir mères célibataires, filles à la morale considérée comme trop légère ou victimes de viol ou d'inceste et, de fait, salies et jugées inaptes à rester dans la société.

En effet, c'est le 25 septembre 1996 que la dernière institution de ce type ferme ses portes en Irlande. Trois ans auparavant, les Sœurs de Notre-Dame de la Charité du Refuge ${ }^{1}$, contraintes pour des raisons économiques de vendre à un promoteur immobilier le terrain sur lequel avait été construite l'une des plus

1. The Sisters of Our Lady of Charity of Refuge. 
grandes institutions du pays, St Mary's Asylum², demandèrent au ministère de l'Environnement un certificat d'exhumation pour les corps de 133 pensionnaires enterrées dans des tombes ne portant aucune inscription ${ }^{3}$.

Lors des exhumations, on découvrit les corps de 22 autres pensionnaires et les religieuses durent demander un certificat d'exhumation supplémentaire. Il apparut plus tard que les Sœurs n'avaient pas pu fournir de certificat de décès pour les 22 femmes concernées et que des identités fictives leur avaient été attribuées. Les corps de 155 femmes furent finalement découverts et ré-enterrés au cimetière de Glasnevin. Des recherches sont actuellement en cours au cimetière de Glasnevin car il semblerait que les noms qui figurent sur les pierres tombales ne correspondent pas toujours au lieu où sont enterrés les corps 4 .

Lexistence, puis la disparition des corps au sein des institutions de type Magdalen Laundries est centrale à toute réflexion sur les modalités de mise en place du régime que James Smith a appelées "Ireland's architecture of containment ${ }^{5}$ ». Cette prise et emprise sur les femmes témoigne du pouvoir exercé par et sur les corps pendant toute la durée de ce que l'on peut appeler le "plein régime " de l'institutionnalisation des femmes en Irlande ${ }^{6}$. Car c'est bien du corps des femmes qu'il s'agit, ce corps sexué, ce corps visible, ce corps tentateur, coupable et responsable qu'il convient de cacher, de désexualiser et de dompter jusqu’à le rendre docile.

Dans un contexte où les sociétés occidentales, et notamment européennes, se voient confrontées à différentes problématiques autour de la liberté et de la visibilité du corps de la femme, et où politiques, média et société civile se saisissent de l'enjeu à des fins diverses, il semble tout à fait pertinent de s'interroger sur la manière dont l'Irlande, au cours de son histoire a reproduit, importé et conçu des espaces et discours destinés à enfermer, châtier et contrôler les corps des femmes. C'est précisément l'objet de cet article, qui tentera de démontrer comment les Magdalen Laundries, institutions totalitaires, héritières du modèle du "grand renfermement ", ont été de véritables fabriques de " corps dociles ".

Ervin Goffman définit ce qu'il appelle une institution totalitaire comme " un lieu de résidence ou de travail où un grand nombre d'individus, placés dans la même situation, coupés du monde extérieur pour une période relativement longue, mènent ensemble une vie recluse dont les modalités sont explicitement et

2. Aussi connu sous le nom de Mary's Penitentiary or Magdalen Home. Institution située à High Park, Drumcondra, qui fonctionna de la fin des années 1850 à 1991.

3. Mary Raftery, "Taking Mary Home”, Irish Times, 15 avril 2004.

4. [https://www.magdalenelaundries.com/name.htm], page consultée le 11 septembre 2016.

5. James M. Smith, Ireland's Magdalen Laundries and the Nation's Architecture of Containment, Notre Dame, Indiana, University of Indiana Press, 2007, p. XIII.

6. On peut considérer que c'est après l'indépendance que ce "plein régime » a été mis en place et qu'il a perduré jusque dans les années 1990. 
minutieusement réglées ${ }^{7}$. Il explique que la prison est un parfait exemple de ce type d'institutions. Les institutions dites totalitaires, selon Goffman, sont caractérisées par " les barrières qu'elles dressent aux échanges sociaux avec l'extérieur, ainsi qu'aux entrées et aux sorties, et qui sont souvent concrétisées par des obstacles matériels : portes verrouillées, murs hauts, barbelés, falaises, étendues d'eau, forêt ou landes ${ }^{8} "$.

Les institutions totalitaires brisent les frontières qui séparent ordinairement les lieux où les individus dorment, se distraient et travaillent. Tous les aspects de l'existence s'inscrivent dans le même cadre. Chaque phase de l'activité quotidienne se déroule en relation de promiscuité totale avec un grand nombre d'autres personnes, soumises aux mêmes traitements et obligations. Toutes ces périodes d'activité sont réglées selon un programme strict, de sorte que toute tâche s'enchaîne avec la suivante à un moment déterminé à l'avance, conformément à un plan imposé d'en haut par un système explicite de règlements dont l'application est assurée par une équipe administrative. Surveillants et surveillés forment un couple indissociable?

Les Magdalen Laundries sont des institutions qui ont évolué au cours du temps, à la fois dans leur structure, leurs objectifs, mais également leur gestion. Les résidentes ${ }^{10}$ des Magdalen Laundries ne furent jamais réellement considérées comme inoffensives, puisque leur existence même était une offense à la société, à ses codes moraux, mais aussi à Dieu. Elles n'étaient pas non plus considérées comme véritablement dangereuses pour la communauté à strictement parler, même si elles l'étaient sur le plan moral. Associés dès le milieu du XIX ${ }^{e}$ siècle à des couvents, et administrés par des ordres religieux, les Magdalen Laundries n'étaient pas destinés à assurer une retraite hors du monde, mais davantage à imposer, à forcer un isolement et une invisibilité. Les caractéristiques communes à ces institutions, telles qu'elles sont définies par Goffman, s'appliquent, en tous points, aux Magdalen Laundries. Il s'agit, en effet, de structures au sein desquelles le quotidien était réglementé de manière stricte, dans le cadre d'un régime autoritaire, et d'un enfermement auquel participaient à la fois "surveillés et surveillants ». La promiscuité qui existait entre les religieuses et les pénitentes conféra à ces institutions un caractère totalitaire dès les années 1930 en Irlande.

7. Erving Goffman, Asiles. Études sur la condition sociale des malades mentaux, Paris, Éditions de Minuit, 1968, p. 41.

8. Ibid., p. 46.

9. Ibid.

10. Notons que les groupes de soutien aux anciennes résidentes utilisent les termes « victims " ou "survivors ». Par souci d'objectivité scientifique et historique, nous choisirons d'utiliser " pensionnaires » ou « résidentes ». 
Pour mieux saisir les enjeux propres à ces institutions, il convient de les replacer dans un contexte historique et philosophique, en s'appuyant sur les théories développées par Michel Foucault.

\section{图 De Foucault à Bentham}

\section{"Le grand renfermement ": misère, assistance et enfermement}

Pour Foucault, c'est l'édit royal du 27 avril 1656 autorisant la fondation d'un Hôpital Général qui signa le premier acte de ce qu’il appelle « le grand renfermement ». Cette structure, parisienne au début, et qui s'étendit par la suite à toutes les villes du royaume, avait pour objectif d'empêcher " la mendicité et l'oisiveté comme les sources de tous les désordres ${ }^{11}$ ». Elle prévoyait que les hommes seraient accueillis à Bicêtre, les femmes à la Salpêtrière, et les enfants à la Pitié. Pour Foucault, les workhouses ou Houses of Correction anglaises s'inscrivaient dans ce schéma, au même titre que les Zuchthäusen allemandes.

Si l'Hôpital Général, selon Foucault, était « une instance de l'ordre, de l'ordre monarchique et bourgeois qui s'organis[ait] en France à cette même époque ", l'Église ne tarda pas, elle aussi, à repenser ses structures d'accueil et à prévoir des institutions susceptibles de concurrencer les entreprises monarchiques. Un vaste réseau caritatif religieux se développa en France au dix-septième siècle. Des hôpitaux ou Charités virent le jour dans de très nombreuses villes, dans lesquels assistance et répression se côtoyaient de près.

Le cas de la Salpêtrière ${ }^{12}$ est tout à fait pertinent pour notre réflexion sur le corps des femmes. Comme nous l'avons mentionné plus haut, cet hôpital faisait partie du projet de l'Hôpital général, conçu par Louis XIV, visant à incarcérer les miséreux et les mendiants.

De quelqu'âge qu'il soient, de l'un et l'autre sexe, qui se trouveront dans la ville et faubourgs de Paris lors de l'établissement de l'Hôpital Général qui ne pourront gagner leur vie seront enfermés dans ledit hôpital et lieux qui en dépendent pour y être employés aux œuvres publiques, manufactures et service dudit Hôpital, selon l'ordre des directeurs ${ }^{13}$.

Au cour de ce schéma, le cas de la Salpêtrière est tout à fait pertinent. On y trouvait toutes sortes de femmes, quelques hommes, souvent âgés, et un très grand nombre d'enfants, assez jeunes, qui avaient suivi leur mère ou avaient été aban-

11. Michel Foucault, Histoire de la Folie à l'âge classique, Paris, Gallimard, 1972, p. 91.

12. Ce nom vient de l'emplacement sur lequel l'établissement fut construit. Il s'agissait, à l'origine, d'une fabrique de poudre à base de salpêtre, destinée aux munitions.

13. Code de l'Hôpital Général, archives de l'Assistance publique - Hôpitaux de Paris, p. 274. 
donnés. Il y avait un grand nombre de " filles enceintes », dont on peut supposer qu'il s’agissait de filles qui avaient été séduites puis abandonnées. Le 20 avril 1684, une nouvelle catégorie de femmes, les "femmes débauchées " firent leur entrée dans l'établissement. Dès 1660, on y comptait 1460 femmes et enfants. En 1701, l'hôpital accueillait 4646 personnes, tous âges confondus, dont 1894 enfants. Les femmes étaient réparties en différentes catégories : les jeunes estropiées et teigneuses, les vieilles paralytiques, les « correctionnaires ", libertines et prostituées et les femmes violentes ou innocentes. À une époque où la moralité était considérée comme primordiale, on enfermait sans aucune limite les prostituées et les femmes dites " gâtées ", qui étaient toutes enceintes. Il s'agissait de femmes dont les grossesses, produits de leur débauche, étaient comparables à une maladie infectieuse ${ }^{14}$.

Tout, dans cette taxinomie, renvoie au corps de la femme, qu'il s'agisse d'infirmités physiques ou de déviance morale. Les corps sont catégorisés et répertoriés pour être mieux soumis et contrôlés. Le terme " femmes débauchées » confere une dimension de jugement moral qui sera au cœur du régime « madeleiniste ${ }^{15}$ » irlandais.

La vie au sein de l'institution était fort semblable au quotidien d'un couvent, les journées étant ponctuées de prières et autres services religieux. Les pensionnaires devaient clairement exprimer une volonté de pénitence et de changement moral. De plus, l'établissement avait une vocation de conversion affichée. Il était indispensable de convertir au catholicisme toutes les femmes impies. Afin de parvenir à ce résultat, et sur le modèle de ce qui se faisait déjà en Hollande, Allemagne et Angleterre, les pensionnaires devaient associer la religion au travail. Le travail forcé devait inculquer une forme de discipline à des individus qui n'en avaient jamais connue. Il est évident que ce que les femmes choisies purent considérer comme une chance n'était ni plus ni moins qu'un moyen, pour les autorités, de se décharger des cas les plus difficiles. C'est précisément la démarche qui sera adoptée par les Magdalen Asylums irlandais.

L'enfermement en France, aux dix-septième et dix-huitième siècles, apparaît donc comme une réponse urgente et, somme tout, peu regardante, à la question de la misère grandissante et du nombre croissant d'indigents. Pourtant, il semble que les femmes aient été particulièrement affectées par ces mesures pénitentiaires. Foucault explique qu'il existe toute une liste d'expériences que le dix-huitième a choisi de bannir.

14. Pour un développement sur le traitement des femmes à la Salpêtrière, voir notamment Carrez, Jean-Pierre, Femmes opprimées à la Salpêtrière de Paris, 1656-1791, Paris, Connaissances et Savoir, 2005; Yannick Ripa, La Ronde des Folles. Femmes, folie et enfermement au XIXe siècle (1838-1870), Paris, Aubier, 1986. Pour une approche artistique de la question, voir Xenakis, Mâkhi, Les Folles d'enfer de la Salpêtrière, Paris, Actes Sud, 2004, ouvrage publié à partir d'une exposition qui eut lieu dans les locaux de l'hôpital.

15. Néologisme. 
Elles touchent toutes, soit à la sexualité dans ses rapports avec l'organisation de la famille bourgeoise, soit à la profanation dans ses rapports avec la nouvelle conception du sacré et des rites religieux, soit au " libertinage " [...]. Ces trois domaines forment, avec la folie, dans l'espace de l'internement, un monde homogène qui est celui où l'aliénation mentale prendra le sens que nous lui connaissons ${ }^{16}$.

La convergence du contrôle de la sexualité et de la moralisation religieuse ne pouvait que conduire à une répression inéluctable dirigée sur les femmes et leurs corps. À défaut de contrôler le corps avant qu'il ne commette l'irréparable, on s'appliquait à re-former des " $\operatorname{corps}_{\text {dociles }}{ }^{17}$ ". Partant de l'endiguement de la misère, et de la répression de la débauche, facteur de désordre social et économique, le pas qu'il restait à franchir vers l'aliénation mentale était assez aisé. Pour Foucault, la sexualité marque la ligne de partage entre Raison et Déraison, ce qui explique pourquoi tant de femmes furent considérées comme "folles " ou " insensées ", en vertu de leur sexualité perçue comme illégale ou d'un rapport différent à leur corps.

En Irlande, la naissance de l'internement des malades mentaux date du dixhuitième siècle ${ }^{18}$. En effet, Jonathan Swift avait légué une partie de sa fortune pour qu'un hôpital spécialisé dans la prise en charge des malades mentaux fût construit.

Il existe peu d'informations, à notre connaissance, sur la condition des femmes dans les institutions pour malades mentaux. Áine McCarthy a publié un article sur l'internement des femmes au sein de l'institution d'Enniscorthy de 1916 à 1925, qui fournit néanmoins quelques indications sur la politique de discrimination qui prévalait ${ }^{19}$. L'article souligne qu'avec le développement des institutions pour malades mentaux, les médecins tentèrent de légitimer les internements par les nouvelles découvertes sur le système reproducteur de la femme. Cette dernière se vit, dès lors, attribuer une série de troubles liés à sa physiologie, notamment la "folie puerpérale ", dont on pensait qu'elle était causée par un empoisonnement dû à l'accouchement, la « folie ovarienne ", résultat d'une inflammation des ovaires, la "folie liée à l'allaitement " et la " mélancolie climactérique ", liée à la ménopause. Ces pathologies rendaient les femmes plus prédisposées à une maladie mentale. Le médecin William Hallaran, qui fut directeur de l'hôpital psychia-

16. Ibid., p. 115-116.

17. Cette expression est tirée de Michel Foucault, Surveiller et Punir, op. cit., p. 13.

18. St Patrick's Hospital ouvrit ses portes en 1746. Voir Malcolm, Elizabeth, Swift's Hospital: a history of St Patrick's Hospital, Dublin 1746-1989, Dublin, Gill and Macmillan, 1989.

19. Áine McCarthy, "Hearts, Bodies and Minds: Gender ideology and Women's committal to Enniscorthy Lunatic Asylum, 1916-1924", in Alan Hayes and Diane Urquhart (eds.), Female Experiences: Essays in Irish Women History, Dublin, Irish Academic Press, 2000, p. 102-109. 
trique de Cork, fut le premier médecin irlandais à écrire un traité sur la folie. En 1818, il expliqua pourquoi les femmes étaient mentalement plus fragiles. "Il ne faut pas s'en étonner lorsque l'on tient compte de tous les facteurs excitants auxquels les femmes sont particulièrement exposées ${ }^{20}$. "McCarthy cite également un extrait d'un manuel de psychologie, datant de 1874, qui confirmait cette prise de parti, affirmant que les femmes dont les organes reproducteurs étaient développés étaient les plus susceptibles d'être atteintes d'hystérie. Cela permit l'internement de nombreuses femmes sous des prétextes relativement fallacieux et ce, jusqu'au milieu du $\mathrm{Xx}^{\mathrm{e}}$ siècle.

L'utilisation de critères pseudo-physiologiques pour justifier une aliénation mentale et par conséquent un internement, allait frapper davantage la femme dont le mode de vie la rendait plus vulnérable, notamment la mère-célibataire, qui représentait un fardeau pour la société et pour sa famille. Elle portait en effet les stigmates les plus évidents d'une sexualité extravertie.

Les pathologies liées à une grossesse et un accouchement difficiles étaient fréquemment associées à l'une des causes de désordre mental mentionnées plus haut, et légitimaient un internement en institutions. La visibilité de la grossesse inscrite sur le corps de la femme pouvait, à elle seule, suffire à prescrire un enfermement.

Ce que nous venons de montrer correspond en tout point aux différents critères cités par Foucault dans sa théorie du "grand renfermement " et nous invite à ne pas envisager le cas de l'Irlande comme particulier. Le corps et la physiologie de la femme offraient une opportunité inespérée de se débarrasser de citoyennes encombrantes et trop voyantes.

En 1975, avec la publication de son ouvrage Surveiller et Punir, Michel Foucault fait découvrir au monde universitaire et académique français l'œuvre considérable de Jeremy Bentham. C'est notamment en analysant le projet panoptique de Bentham qu'il ouvre la voie à une analyse poussée du projet de ce juriste et philosophe utilitariste, qui avait conçu tout un arsenal de méthodes censées résoudre le problème de l'assistance aux pauvres, alors que se dessinaient les contours d'une réforme de cette assistance en Angleterre.

\section{«Corps dociles " et corps de femmes}

Selon Foucault, à la fin du dix-huitième siècle et au début du dix-neuvième, l'on assiste à une transformation de la punition. Il distingue deux grands mouvements : d'une part, "l'effacement du spectacle punitif ${ }^{21}$ ", avec une tendance

\footnotetext{
20. "This cannot be wondered at when we take into account the many exciting causes to which females are more particularly exposed ", W. H Hallaran, Practical observations on the Causes and Cure of Insanity, Cork, Edwards and Savage, 1818, p. 50.

21. Michel Foucault, Surveiller et Punir, Paris, Gallimard, 1975, p. 15.
} 
à cacher l'expression de l'acte punitif. Ce dernier consiste à " corriger, redresser, guérir $^{22} »$. Et d'autre part, un recul devant l'utilisation du corps comme lieu de la punition et de la répression.

Le corps s'y trouve en position d'instrument ou d'intermédiaire : si on intervient sur lui en l'enfermant, ou en le faisant travailler, c'est pour priver l'individu d'une liberté considérée à la fois comme un droit et un bien. Le corps, selon cette pénalité, est pris dans un système de contraintes et de privation, d'obligation et d'interdits ${ }^{23}$.

Cette notion est d'autant plus pertinente et importante lorsque c'est le corps lui-même et notamment celui de la femme, qui est à la source du châtiment et de la punition. Pour autant, nous dit Foucault, la prise sur le corps n'est pas complètement révolue, puisque l'enfermement contient, en soi, un "supplément punitif qui concerne bien le corps lui-même : rationnement alimentaire, privation sexuelle, coups, cachot $^{24}$ ". Ainsi, ce serait à l'âme, et non plus au corps que s'adresserait la pénalité. Foucault ne le croit fondamentalement pas. Il s'en explique en arguant que " c'est bien toujours du corps qu'il s'agit - du corps et de ses forces, de leur utilité et de leur docilité, de leur répartition et de leur soumis$\operatorname{sion}^{25}$. Le corps s'inscrit dans le système complexe des rapports de production, de pouvoir et de domination, et il n'est « force utile que s'il est à la fois corps productif et corps assujetti ${ }^{26}$ ". La discipline, le contrôle, le pouvoir et la surveillance ont pour objet de forger des " corps dociles " : "La discipline fabrique ainsi des corps soumis et exercés, des corps "dociles"27."

Sa théorie s'est appliquée, de tout temps, aux femmes en tant que catégorie spécifique, a fortiori parce que leur corps était objet et source de pouvoir. L'illégitimité, notamment, était perçue comme la trace " corporelle " d'une utilisation frauduleuse du corps. Ce corps de femme avait produit, dans l'illégalité, un ou plusieurs autres corps, et c'était ces corps mêmes qu'il fallait contrôler et punir. C'était bien sur eux que devaient s'exercer les « technologies du pouvoir " dont parle Foucault.

Dans Surveiller et Punir, il affirme que toutes les instances de contrôle individuel, qu'il s'agisse des hôpitaux, des asiles et des prisons, ont opéré un partage entre le normal et l'anormal, un "marquage binaire ». 
Le Panopticon ${ }^{28}$ de Bentham, dit-il, « est la figure architecturale de cette composition $^{29}$ ». Ce que Foucault voit dans le panoptisme, c'est la possibilité, pour les instances du pouvoir, de contrôler en permanence, d'" induire chez le détenu un état conscient et permanent de visibilité qui assure le fonctionnement automatique du pouvoir ${ }^{30} »$.

Ce qui est intéressant dans l'analyse qu'en fait Foucault, c'est la double dimension du projet disciplinaire. Il s'agissait de contrôler, de surveiller, de réformer, et de réajuster un mode de fonctionnement en accord avec une certaine idéologie du pouvoir.

C'est cet aspect qui nous semble tout à fait pertinent dans l'analyse de l'évolution des différents modes de prise en charge de la « femme perdue » en Irlande du milieu du dix-neuvième au milieu du $\mathrm{xx}^{\mathrm{e}}$ siècle. Il interroge, en effet, la nature et la forme de discipline envisagées, le type de pouvoir auquel elle correspondait et le degré d'emprise, ou de prise, sur le corps qu'elle prévoyait.

Plus intéressant encore, Bentham se prononça sur les institutions de prise en charge des prostituées qui existaient à l'époque en Angleterre. "Les hôpitaux établis à Londres pour les filles repentantes sont de bonnes institutions, mais ceux qui condamnent fermement la prostitution ne sont pas en accord avec eux-mêmes s'ils encouragent ces œuvres caritatives. Si elles remettent certaines filles dans le droit chemin, elles en encouragent d'autres ${ }^{31}$."

Il faisait référence, indubitablement, aux ancêtres des Magdalen Asylums, établis en Angleterre au cours du dix-huitième siècle par des philanthropes protestants ${ }^{32}$.

Sherill Cohen qui a publié un ouvrage sur l'histoire des institutions pour femmes de 1500 à $1992^{33}$ affirme, parlant de Foucault et de son analyse du projet panoptique de Bentham, que pour les femmes, la société patriarcale occidentale a toujours été " un régime panoptique ${ }^{34}$ ».

28. Néologisme fabriqué par Bentham pour rendre compte de la structure de totale visibilité qu'il envisageait. Le terme français panoptique fut utilisé dans le document présenté à l'Assemblée nationale en 1791 : J. Bentham, Panoptique. Mémoire sur un nouveau principe pour construire des maisons d'inspection, et nommément des maisons de force, E. Dumont (éd.), Paris, Imprimerie nationale, Secours publics nº 1, 1791.

29. Michel Foucault, Surveiller et Punir, op. cit., p. 233.

30. Ibid., p. 234.

31. "The hospitals established in London for repentant girls are good institutions: but those who regard prostitution with absolute rigour are not consistent with themselves, when they approve of these charitable foundations. If they reform some, they encourage others", Jeremy Bentham, The Works of Jeremy Bentham, published under the Superintendence of his Executor, John Bowring (Edinburgh, William Tait, 1843). 11 vols. Vol. 1, [http://oll.libertyfund. org/title/2009/140060/2643427], page consultée le 21 septembre 2007.

32. On peut, à ce titre, citer le Lock Hospital (1746) pour les vénériennes et le Magdalen Hospital (1758), pour prostituées, tous deux situés à Londres.

33. Sherill Cohen, The Evolution of Women's Asylums since 1500: from Refuges for ex-prostitutes to Shelters for battered women, New York, OUP, 1992.

34. Ibid., p. 5-6. 
Les institutions pour femmes européennes des seizième et dix-septième siècles furent d'importants archétypes pour certaines institutions spécialisées contemporaines visant à prendre en charge un seul sexe, à savoir les femmes. Plus généralement, l'histoire des institutions pour femmes est également liée au développement d'établissements correctionnels, éducatifs et caritatifs réservés à la plèbe au cours des siècles suivants. Pour cette raison, l'histoire des refuges pour femmes peut offrir de nouvelles perspectives sur la nature des institutions sociales modernes ${ }^{35}$.

Ce sont précisément les philanthropes anglais qui ont importé le modèle européen et qui l'ont exporté vers l'Irlande. Les modalités et objectifs ont évolué avec le temps mais également avec le discours et les représentations identitaires et religieuses.

\section{Corps en prison, prison des corps}

\section{Investir le corps}

Jonas Hanway, membre d'un groupe de philanthropes qui fondèrent le Magdalen Hospital de Londres ${ }^{36}$, était un marchand qui avait parcouru le monde et avait observé avec attention le fonctionnement des entreprises caritatives dans le reste de l'Europe. Il rédigea plusieurs pamphlets vantant les mérites d'un Magdalen Hospital à Londres. Dans l'un deux, il revient sur les expériences des nations étrangères pour justifier sa nouvelle entreprise : "J'ai lu de nombreux ouvrages sur la question et fait plusieurs enquêtes, et je constate que la même formule a été adoptée dans la plupart des pays chrétiens dans le monde ${ }^{37}$. " Il décrit les institutions hollandaises et les ordres monastiques appelés Magdalanes, spécifiquement consacrés à la prise en charge des prostituées repentantes. Ces ordres avaient été établis entre le treizième et le seizième siècle à Marseille, Metz, Naples, Paris, Rouen et Bordeaux, ainsi qu’en Espagne ${ }^{38}$.

Voici ce qu'il écrit à propos de l'institution qu'il compte fonder : " [Cet établissement] sera un moyen d'employer les oisives, de leur enseigner et de les habituer

\section{Ibid.}

36. Cf. note 28, p. 11.

37. "I have turned over some volumes on the subject, and made several enquiries, and I find the same thing has been adopted by almost every Christian country in the world", Jonas Hanway, Reflections, Essays and Meditation on Life and Religion, London : printed for John Rivington. R. and J. Dodsley, and C. Henderson, 1762, p. 8-9.

38. L’Hôpital du Refuge fut fondé à Marseille en 1640, le Refuge de Clermont en 1668. Le couvent de NotreDame de la Charité du Refuge, l'une des institutions les plus importantes, vit le jour à Caen en 1641, et prit ensuite le nom de Bon-Pasteur. L'ordre avait été fondé par Jean Eudes. Dans l'ensemble, les institutions françaises étaient plus dures et plus répressives que leurs homologues italiennes. 
à travailler; de réformer leur morale; de sauver de nombreux corps de la maladie et de la mort, et de nombreuses âmes d'une misère éternelle ${ }^{39}$. "

On peut constater que le corps apparaît déjà comme l'espace dans lequel vont se mettre en œuvre les technologies du pouvoir. L'objectif est, certes, moral mais c'est par le corps que passera la rédemption. Le corps de la prostituée représentant son outil de travail, il fallait lui retirer, ne fut-ce symboliquement, cet outil, en désexualisant son corps. Il faut aussi garder à l'esprit que les «femmes déchues ${ }^{40}$ " représentaient également un danger physique, puisque l'on craignait la transmission de maladies vénériennes.

$\mathrm{Si}$, au XVIII ${ }^{\mathrm{e}}$ siècle, ces institutions avaient pour vocation de mettre à l'abri des femmes qui avaient succombé au péché de chair et se trouvaient dans une situation délicate, elles ont évolué, au cours du dix-neuvième siècle vers des établissements punitifs, des institutions quasi-totalitaires. Les durées de séjours sont devenues de plus en plus longues et les formations à un métier en vue d'une réinsertion dans la société ont progressivement été remplacées par un dur labeur systématique censé financer l'institution. Le travail de blanchisserie et sa pénibilité sont devenus le symbole de la purification morale et physique dont on estimait que les femmes devaient s'acquitter pour faire acte de pénitence.

Bien que les prémices du "madeleinisme " en Irlande aient été de nature philanthropique et laïque, petit à petit, faute de financement, et l'enthousiasme des bienfaiteurs ayant diminué, la plupart des institutions ont été confiées à des ordres religieux, leur conférant une nouvelle dimension. Quoi qu'il en soit, ce sont les femmes, qu'elles soient philanthropes ou religieuses, qui ont dominé ce mouvement. Il s'agissait au début de protestantes issues de la bourgeoisie, puis de religieuses catholiques. Ce glissement a opéré un changement considérable dans la manière dont les femmes déchues étaient perçues et traitées. L'humanisme indulgent du dix-huitième a progressivement cédé la place à une culpabilisation impitoyable, reléguant les aspirations réhabilitatrices des origines au second rang pour les remplacer par un régime punitif et répressif. La femme déchue n'était, dès lors, plus envisagée comme une victime mais comme une coupable, et la compassion qui s'exprimait notamment à l'égard des mères célibataires dans les premières institutions a disparu avec l'entrée sur la scène madeleiniste des religieuses.

39. "It will be a means of employing the idle, of instructing them in, as well as habituating them to work; of reforming their Morals; of rescuing many bodies from Disease, and Death; and many Souls from eternal Misery ", Jonas Hanway, op. cit., p. 31-33.

40. Traduction du terme "fallen women " employé en anglais. 


\section{Corps disciplinés}

L'un des aspects qui préoccupait beaucoup le gouvernement et l'Église Catholique en Irlande pendant les années 1920 et 1930 fut le cas de la mère-célibataire. Selon l'idéologie du jeune État, fortement influencé par la doctrine sociale de l'Église, la place de la femme était au foyer, et la femme idéale était la Mère. La femme devint emblématique du discours de construction identitaire de l'État Libre. Alors que la maternité était idéalisée dans la rhétorique politique ${ }^{41}$ de l'Irlande, le corps de la mère qui n'appartenait pas à ce schéma idéal fit l'objet d'une attention particulière et d'un grand nombre de discours politiques et religieux.

La sexualité était au cour des préoccupations de l'Irlande libre mais c'était pour mieux la cacher. On parlait sans cesse de sexe mais pour dire qu'il ne fallait ni en parler, ni y penser, et n'y avoir recours que pour procréer. Elle opérait le partage entre pur et impur, moral et immoral, normal et transgressif.

Le travail, notamment de blanchisserie, mais également de couture, était à la fois une source de revenus pour les religieuses mais également symbolique d'une technique de purification. La pénibilité du travail imposait au corps le châtiment qui revenait à l'âme. Une discipline de fer régnait, sous la surveillance permanente des religieuses. Les femmes étaient peu nourries, dormaient peu, travaillaient sans relâche, dans des conditions qui sont désormais condamnées par les instances internationales comme du travail forcé et de la quasi-torture, comme nous le verrons plus loin. Des témoignages ${ }^{42}$ relatent des accidents, des brûlures non soignées, des heures passées sans boire ni manger, dans les vapeurs de produits toxiques. Si une pensionnaire était malade, elle restait au lit quelques jours sans que l'on fasse venir un médecin.

On surveillait et on ne tardait pas à punir celles qui ne respectaient pas les règles. Des corps "purs ", ceux des religieuses, côtoyaient des corps "impurs ", ceux des femmes dites perdues. La symbolique de Marie et celle de Marie-Madeleine étaient enfermées dans le même espace, soumises à un même régime d'exclusion du monde. Le corps était non seulement malmené et maltraité mais il était également négligé, oblitéré.

Dès leur entrée dans l'institution, les femmes se voyaient retirer leur nom et attribuer un nom de sainte. On leur coupait les cheveux, signe ostentatoire de

41. Pour une analyse de cette question, voir Maryann Valiulis, « Neither feminist Nor Flapper : the Ecclesiastical Construction of the Ideal Irish Woman ", in Mary O'Dowd and Sabine Wichert (eds.), Chattel, Servant or Citizen? Women's Status in Church, State and Society, Belfast, Institute of Irish Studies/QUB, 1995, p. 168-178.

42. Pour les témoignages des ancienne pensionnaires, voir : [http://www.magdaleneoralhistory.com]; Sex in A Cold Climate, documentaire de Steve Humphries, réalisé en 1997; Nancy Costello, Kathleen Legg, Diane Croghan, Marie Slattery and Marina Gambold with Steven O'Riordan, Whispering Hope:The True Story of the Magdalene Women Orion, UK, 2015; le rapport de la Commission dite McAleese : Report of the Inter-Departmental Committee to establish the facts of State involvement with the Magdalen Laundries (2013). 
féminité et de vanité. On leur donnait une sorte de vêtement informe, d'une couleur indéfinie. Rien ne devait donner à voir ce corps, responsable de leur état de disgrâce, qui était pourtant l'objet de toutes les attentions.

La plupart des pensionnaires étaient des femmes qui avaient mis au monde un enfant dans le péché. Elles étaient passées par une autre institution, le Mother and Baby Home, où elles avaient accouché, puis, bien souvent, leur enfant leur avait été retiré pour être adopté ou placé dans un orphelinat ou une Industrial School. La violence de l'arrachement du bébé, à peine sevré, était une autre atteinte à l'intégrité du corps, le corps maternel ${ }^{43}$.

Ces dispositifs et technologies de pouvoir - la pénibilité du travail, la dissimulation de la féminité, la violence faite au corps maternel et le silence imposé aux pensionnaires, furent suffisants pour insuffler un climat de peur tel qu'il ne fut pas nécessairement utile pour les religieuses d'avoir recours à la violence physique, ce qui ne fut malheureusement pas le cas dans les institutions qui accueillirent des enfants. L'obéissance et la docilité étaient le produit de la crainte et d'une discipline quotidienne et répétitive, dans un schéma panoptique où les pensionnaires se savaient en permanence sous contrôle.

Dans les Magdalen Laundries, le corps est l'instrument par lequel passe la purification de l'âme. C'est en soumettant le corps que l'âme pourra être sauvée. Il s'agit d'une technique très pernicieuse puisque le corps devient également l'instrument qui produit, qui travaille. Il est l'outil qu'il convient de ménager afin qu'il puisse rester efficace mais également celui du châtiment et, par conséquent, il fait l'objet de maltraitance.

Dans le même temps, la femme voit son corps disparaittre derrière des discours d'immoralité et devenir l'instrument par lequel passe sa prétendue rédemption.

\section{Corps dociles?}

Lorsque les médias ont révélé l'ampleur du régime d'institutionnalisation des femmes vulnérables en Irlande, la parole s'est libérée, des témoignages ont dénoncé les violences subies et les traumatismes. Les anciennes pensionnaires ont eu la plus grande difficulté à se réinsérer dans la société. Leur parcours témoigne de souffrances psychologiques et physiques. Leur corps porte la mémoire d'années de privations, de confinement, d'isolement, de manque de soins et parfois d'abus sexuels.

Il n'en demeure pas moins que grâce au travail et au soutien d'associations de victimes, un processus de justice et réparation est actuellement en cours ${ }^{44}$. Comme

43. Ces institutions font à leur tour l'objet d'une Commission d'Enquête : [http://www.mbhcoi.ie/MBH.nsf/page/ index-en].

44. Voir le site de JFMR à ce sujet. 
signalé plus haut, des recherches sont actuellement menées par JFMR afin de tenter de localiser précisément les lieux ou sont enterrées les femmes. Cela permettra de rendre une certaine forme d'intégrité aux corps des défuntes.

De plus, le groupe de soutien $J F M R^{45}$ a mené sans relâche une campagne pour obtenir des autorités politiques la reconnaissance des souffrances endurées ${ }^{46}$, ce qui a conduit à la mise en place de la Commission McAleese ${ }^{47}$. Le groupe a également déposé une requête auprès du Comité des Nations Unies Contre la Torture (UNCAT) ${ }^{48}$ pour incarcération forcée, travail forcé, mauvais traitements, en vertu des articles 1 et 16 de la Convention contre la Torture ${ }^{49}$.

En figurant au cœur de la campagne pour obtenir justice et réparation, le corps est extrait de son état passif et docile. Le silence qui lui a été imposé est désormais remplacé par des mots, actes performatifs, qui lui restituent une visibilité qu'il ne saurait être question d'ignorer plus longtemps.

\section{圈 Conclusion}

Si le processus d'institutionnalisation de milliers de femmes en Irlande dans les Magdalen Laundries a participé d'un mouvement général historique d'enfermement des individus jugés transgressifs, il n'en demeure pas moins que le pays a développé des techniques de pouvoir et de contrôle qui lui ont été propres, adossées à un discours de construction identitaire très influencé par la doctrine sociale de l'Église catholique.

L'Irlande s'est incarnée dans un ordre moral, régi par un appareil législatif strict et rigide, une idéologie sociale dans laquelle la femme était à la fois symbolique de la pureté de la nation et emblématique de toutes les transgressions possibles. À ce titre, les Magdalen Laundries incarnent ce régime dans lequel les corps ont été investis et travestis.

La récente légalisation du Mariage Pour Tous et la campagne menée pour l'abolition du $8^{\mathrm{e}}$ amendement de la Constitution, portant sur l'illégalité de l'avortement, témoignent d'une volonté de réincarner le pays, à l'aune d'une vision sociétale qui restitue au corps sa dimension légitime.

45. [http://www.magdalenelaundries.com/what.htm]. Page consultée le 12 septembre 2016.

46. 'https://www.magdalenelaundries.com/JFMR\%20Followup\%20UNCEDAW\%20300915.pdf]. Page consultée le 12 septembre 2016.

47. [http://www.justice.ielen/JELR/Pages/MagdalenRpt2013]. Page consultée le 12 septembre 2016.

48. [http://www.magdalenelaundries.com/jfm_comm_on_torture_210411.pdf]. Page consultée le 12 septembre 2016. 49. [http://www.ohchr.org/FR/ProfessionalInterest/Pages/CAT.aspx]. Page consultée le 13 septembre 2016. 